\title{
Extractable and Leachable Testing in Pharmaceutical Analysis
}

Zhong $\mathbf{M}^{*}$

PharmaForce Inc., United States

*Corresponding author: Meng Zhong, R\&D Senior Scientist, PharmaForce Inc., A Subsidiary of Luitpold Pharmaceuticals Inc., Ohio, United States, E-mail: mzhong@pharmaforceinc.com

Received date: May 29, 2018; Accepted date: May 30, 2018; Published date: June 06, 2018

Copyright: (c) 2018 Zhong M. This is an open-access article distributed under the terms of the Creative Commons Attribution License, which permits unrestricted use, distribution, and reproduction in any medium, provided the original author and source are credited.

\section{Editorial}

Pharmaceutical packaging materials serve as the first line of defense to ensure drug quality; however, the use of the material itself potentially possesses a risk of drug contamination. In recent years, increased concern surrounding extractables and leachables (E\&L) in pharmaceutical drug products have been raised by the U.S. Food and Drug Administration (FDA) and European Medicines Evaluation Agency (EMEA). The primary concern of E\&L evaluation is the public health and safety, as these chemicals could have toxicity, immunogenicity or potentially interactions with the active ingredients in the product. Additionally, these chemicals could lead to drug efficacy loss or a decrease in drug stability during storage [1]. Furthermore, the effects of these chemicals on biologics are unknown and unpredictable. The possible effects include biological molecules folding, state changes, aggregation, oxidation, formation of particulate matter etc.

Regulatory agencies worldwide provide formal and comprehensive documents explaining the evaluation of E\&L in pharmaceutical products. In 1999, the FDA published the first guidance for container closure systems for Human Drugs and Biologics. Pharmaceutical manufacturers are expected to demonstrate the safety of the materials involved in the packaging system [2]. In 2014, two new chapters USP $<1663>$ and $<1664>$ were published by the United States Pharmacopiea (USP) regarding the design, execution, and justification of the E\&L.

The terms "Extractable" and "Leachable" are defined in USP <1663> and $<1664>$. In short, Extractables are organic and inorganic chemicals released from packaging material or delivery systems using extraction solvents under laboratory conditions [3]. Analysis of extractables is often performed by exposing the packaging component to solvents covering a broad range of polarity, $\mathrm{pH}$, and ionic strength. It is normally conducted at elevated temperatures using techniques such as reflux, soxhlet, sonication, etc. The aim is to mimic the pharmaceutical formulation, yet create a worst-case scenario using extreme conditions to force the release of any possible extractables. Leachables are chemicals present in the drug product that are from their contact with packaging materials or during manufacturing process under recommended storage conditions or in the accelerated stability program [4]. Leachable analysis is conducted using the actual formulation in its final container closure system, including both primary and secondary packaging under normal conditions. Typically, leachables are a subset of extractables or are derived from extractables. Otherwise, it may suggest the extraction study is not aggressive enough to cover all the possibilities [5]. In general, an extractable-leachable correlation will be established to evaluate the likelihood and the quantity of potential leachables to be present in the final product using data from the simulated extraction studies.
Extractable/Leachable evaluation is a critical step in the development of pharmaceutical products, especially the inhaled and nasal drug product (OINDP), parenteral and ophthalmic drug products (PODP), and transdermal pharmaceutical products; which are considered as high risk. It is not only the final container closure system that needs to be considered, as materials used in the manufacturing process are equally important. Manufacturing equipment (Tanks, tubing, Diaphragms, filters, fillers, etc.), plastic and elastomeric components (vials, stoppers, prefilled syringes, and plastic premix bags), inks and adhesives from labels, and degradation products are all potential sources of E\&L. Unfortunately, the assessment cannot be performed using one single method as the sample matrix can be quite complex. Thus, the potential E\&Ls are grouped into three categories based on their chemical properties, and the corresponding test methods are listed, following industrial standards [5].

\section{Volatile Organics and Semi-volatile Organics}

Volatile Organic Compounds can be analyzed by Gas chromatography-Mass Spectrometry (GC-MS), while semi-volatile organics can be tested by Head Space GC-MS. It is often performed using a predominantly non-polar capillary column with a broad range temperature scan. The EI (Electron Ionization) spectra are used for the identification of the individual extractable and leachable, through the computerized library search. Gas chromatography-Flame Ionization Detector is used in lab screening and quantitative method for its general accessibility and low cost. Additionally, for N-nitrosamines, a general component in rubber manufacturing and a carcinogenic, Gas chromatography-Terminal energy analysis detector (GC-TEA) is also used taking advantage of its sensitivity level at the low ppb range.

\section{Polar, Non-Volatile}

The common technique to detect and quantify the non-volatile compounds are Liquid chromatography-Mass Spectrometry (LC-MS) and Liquid chromatography-Diode array detector (LC-DAD). Similar to GC-FID, LC-DAD is often used as a routine extractable testing and general lab screening method. Reversed phase LC is commonly used, scanning from low to high organic solvent gradient. The MS identification is more difficult than GC-MS since the computerized library is not readily available. In general, polymer stabilizers and antioxidants (e.g. Irganox 1010 or Diphenylamine) are analyzed by LC techniques.

\section{Inorganic}

Inorganic E\&Ls, mostly metals, are analyzed by Inductively Coupled Plasma-Mass Spectrometry (ICP-MS), Inductively Coupled Plasma- 
Citation: Zhong M (2018) Extractable and Leachable Testing in Pharmaceutical Analysis. Pharm Anal Acta 9: e193. doi:

Page 2 of 2

Optical Emission Spectroscopy (ICP-OES), Graphite Furnace Atomic Absorption Spectroscopy (GF-AAS) or Energy Dispersive X-ray (EDX). For analytes with high quantity, GF-AAS is an economical choice for both detection and quantitation. EDX is a qualitative method using dried residue of extracts. ICP coupled method offered great sensitivity in analyzing trace amount of inorganic E\&Ls. Samples are often digested using aqueous solutions to be compatible. The common solvent for the extraction study is nitric acid solution.

The complexity of the compounds of interest can lead to lengthy and costly studies. It is the responsibility of analytical scientists to understand the type of packaging materials, the drug formulation, and the route of administration to decide the most realistic studies. A reliable E\&L identification method and an accurate quantitative method set the foundation for drug product safety impact assessments.

\section{References}

1. Jenke D (2007) Evaluation of the chemical compatibility of plastic contact materials and pharmaceutical products; safety considerations related to extractable and leachables. J Pharm Sci 96: 2566-2581.

2. Markovic I (2007) Evaluation of safety and quality impact of extractable and leachable substances in therapeutic biologic protein products: a riskbased perspective. Expert Opin Drug Saf 6: 487-491.

3. Assessment of Drug Product Leachables Associated with Pharamceutical Packaging/Delivery System (2013) USP General Chapter 1663.

4. Assessment of Drug Product Leachables Associated with Pharamceutical Packaging/Delivery System (2013) USP General Chapter 1664.

5. Norwood DL, Paskiet D, Ruberto M, Feinberg T, Schroeder A, et al. (2008) Best practices for extractable and leachables in orally inhaled and nasal drug products: An overview of the PQRI recommendations. Pharm Res 25: 727-739. 